\title{
ACCELERATION BEHAVIOR OF DRIVERS IN A PLATOON
}

\author{
Ghulam H. Bham \\ Rahim F. Benekohal \\ Department of Civil Engineering \\ University of Illinois at Urbana-Champaign \\ Urbana, IL, USA \\ E-mail: bham@uiuc.edu \\ E-mail: rbenekoh@uiuc.edu
}

\begin{abstract}
Summary: A new dual-regime acceleration model was developed to represent the acceleration behavior of drivers in a platoon of vehicles. Two sets of field data collected by aerial photographic techniques were used to assess the validity of the proposed and existing acceleration models. A single regime acceleration model failed to present the acceleration behavior of drivers. The field data indicated that at around $13 \mathrm{~m} / \mathrm{sec}$ the acceleration rate drops. Thus, two different acceleration rates, higher acceleration rate at lower speeds and lower acceleration rate at higher speeds, were used to provide the best fit to the data. This provided realistic acceleration behavior of drivers in a platoon. The field data sets were collected about 10 years apart. The improvements in acceleration capability of a platoon of vehicles from two different time periods were determined. Improvements in performance of vehicles were quantified using the above mentioned field data. The method of quantification can also be used to predict and model the performance of vehicles currently in use. Inversely, current vehicles can be downgraded to represent vehicles of past years and thus make use of already collected data. Important uses of the dual regime model are in modeling the traffic flow behavior and designing roadway elements that depend on acceleration behavior of drivers.
\end{abstract}

\section{INTRODUCTION}

The modeling of driver behavior and vehicle characteristics is of fundamental importance in traffic engineering for design of roadway elements and modeling of traffic flow. One of the main characteristics of a vehicle concerning traffic and highway engineers is its acceleration capability. Acceleration capability is important in several traffic operations, such as passing maneuvers, intersection design, sight distance and gap acceptance. In addition, the dimensioning of highway features such as freeway ramps and passing lanes is often governed by acceleration rates. Errors in modeling acceleration capability of vehicles would lead to inappropriate design of highway features, which could lead to accidents, resulting in loss of life and property.

The paper describes the modeling of acceleration behavior of drivers in a platoon. It examines the single-regime model (constant acceleration model), based on which it proposes a dual-regime model, which better fits the acceleration behavior of drivers. The paper is a valuable reference for practitioners, as it explains the limitations of using the single-regime model. In addition, researchers involved in the development of traffic simulation models, and modeling driver and acceleration behavior of vehicles can benefit from it. 


\section{FIELD DATA SETS}

Two data sets were used to study acceleration behavior of drivers in a platoon. One data set was from 1968 and the other from 1983. The data sets were used to study the performance of vehicles and the change in acceleration capabilities of vehicles over the years. The vehicular characteristics are then used in modeling the behavior of drivers in a platoon. The data sets were the Ohio State University Data (OSU data) (Treiterer, 1975) and the data collected by the FHWA (Smith, 1985). Both data sets were collected using aerial photographic techniques and provide speed and position for all vehicles at one-second intervals. In the OSU data, speed and position of 13 vehicles in a platoon was captured and is estimated to be accurate within $\pm 0.15 \mathrm{~m}$ and \pm 1.6 $\mathrm{km} / \mathrm{h}(1 \mathrm{mph})$, respectively. The OSU data is for platoon123 that traveled on one lane of I-70 near Columbus, Ohio. The FHWA data was collected at Mulholland Drive in Los Angeles, California. The data set is accurate within 0.9 to $1.5 \mathrm{~m}$. Data of 12 vehicles in a platoon traveling on the left most lane of a 5-lane freeway is used in the analysis. Both data sets comprise of passenger vehicles only.

For both data sets, the speed of vehicles accelerating from a stopped position was captured. The speed of vehicles was averaged out at every time second as if they accelerated at the same time. This provided an average speed profile for all vehicles in acceleration. The average speed profile of vehicles for the OSU and FHWA data is shown in Figure 1. The speed profiles represents the average normal acceleration behavior of vehicles accelerating from a stopped position. From these profiles, the parameters for the single and the dualregime models were acquired. This is explained in the section on Acceleration Modeling.

\section{VEHICLE PERFORMANCE}

The acceleration behavior varies for different drivers, surface conditions, and for different types of vehicles; passenger cars showing different acceleration rates compared to buses and trucks. In Figure 1, the FHWA data shows an increase in performance of approximately $28.1 \%$ over vehicles in the OSU data. Additional proof of improved performance of vehicles over the years is presented in Figure 2, which
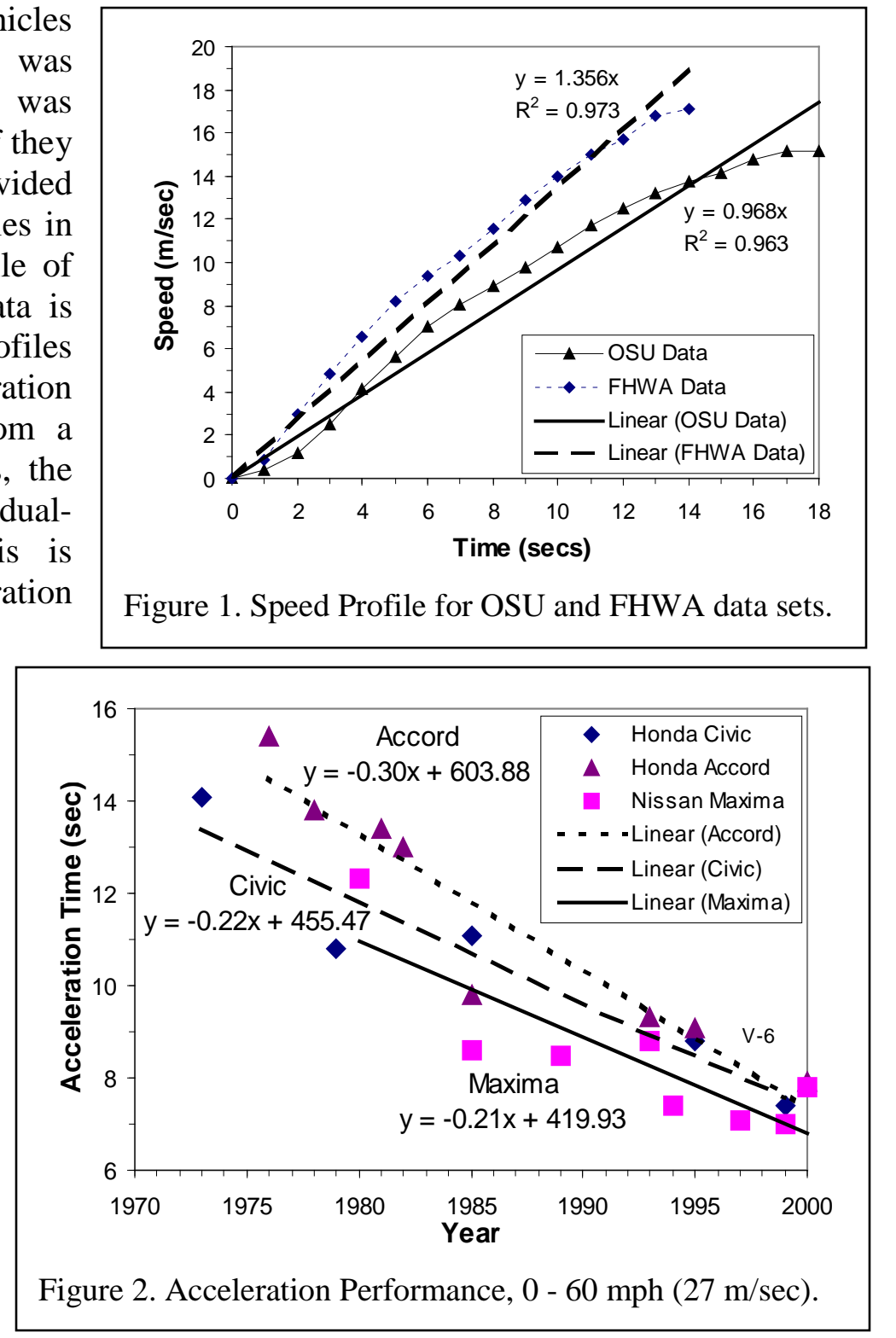
shows the maximum acceleration capabilities of sedans. Acceleration characteristics of different brands of sedans were noted mainly from Road and Track magazine ${ }^{\circledR}$. Data from different years were collected so as to show the trend and change in acceleration performance. The acceleration behavior shows improvement over the 30 years of data shown. The size of vehicles and types of engines have changed as well, but this is not discussed and not an issue in this paper.

Models like Honda's Accord and Civic, Nissan's Maxima were chosen based on their popularity and share in the auto market. These vehicles represent normal sedans in road traffic. SUV's, sports cars, passenger vans and trucks were not considered, since the data is only shown here to indicate the trend in vehicle performance. Linear regression models were used to fit the vehicular performance data shown in Figure 2. Average acceleration times for each year, from the linear regression models for Accord $\left(\mathrm{R}^{2}=0.90\right)$, Civic $\left(\mathrm{R}^{2}=0.91\right)$, and Maxima $\left(\mathrm{R}^{2}=0.72\right)$ were then used to plot and extrapolate average acceleration times $(0-60 \mathrm{mph})$. This is presented in Figure 3.

From Figure 3, the 0-60 mph time for sedans from 1968 and 1983 was found to be 14.87 and 11.22 seconds, respectively, an acceleration ratio of 1.32 and which shows an improvement in acceleration performance of $24.51 \%$ in 15 years. This is approximately equal to the improvement shown by FHWA data over OSU data collected around the same years. This technique presents an interesting way to predict the improvement in acceleration performance of vehicles in the future using the $0-60 \mathrm{mph}$ data. Similarly the technique can be utilized to make use of data collected in the past, by degrading the acceleration capability of today's vehicles. This will provide means to make good use of older data that can otherwise be useless.

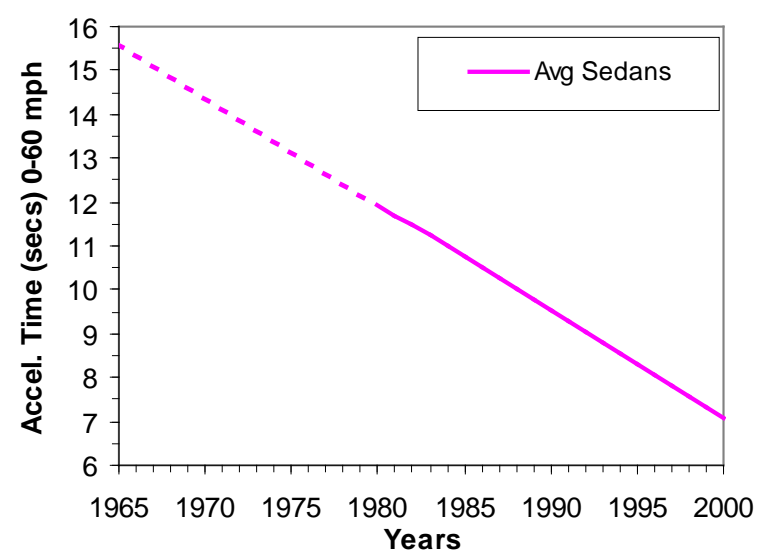

Figure 3. Average acceleration time, showing trend for sedans (0-60 mph).

\section{ACCELERATION MODELING: SINGLE-REGIME MODEL}

The single-regime acceleration model is the simplest and the most commonly used acceleration model. It uses a constant acceleration rate for vehicles accelerating from an initial speed to their final speed. The constant acceleration rate is the average rate of acceleration and can be calculated in a number of ways, as follows:

$$
a_{\text {avg }}=\frac{2 x}{t^{2}}=\frac{v_{f}^{2}-v_{i}^{2}}{2 x}=\frac{v_{f}-v_{i}}{t}
$$

where

$$
\begin{aligned}
& a_{a v g}=\text { average acceleration rate, } \mathrm{m} / \mathrm{sec}^{2} \\
& x=\text { distance covered in time } t \text {, meters } \\
& t=\text { time required to cover distance } x \text {, or reach a velocity of } v_{f} \text { from } v_{i} \text {, seconds } \\
& v_{f}=\text { velocity at time } t, \mathrm{~m} / \mathrm{sec} \text {, and } \\
& v_{i}=\text { starting velocity, } \mathrm{m} / \mathrm{sec} \text {. }
\end{aligned}
$$

However, when field data is available, acceleration rate can be calculated by fitting a linear regression line through the data points. This is shown in Figure 1. In addition, it can be observed 
in Figure 1, that the ratio of average acceleration rates for the data sets equals $1.4(1.356 / 0.968)$. This is very close to the value of 1.32 obtained from Figure 3 for the years 1968 and 1983, when the data sets were collected. This shows the usefulness of the technique discussed above, in predicting the performance of vehicles in the future using current 0-60 mph commonly available data.

Although, the single-regime acceleration model is the most commonly used model, it doesn't represent realistic acceleration capability of vehicles, even with the high R-squares. This is also evident from the fit of the single-regime model to the field data sets shown in Figure 1 . The model is erroneous since it assumes a single acceleration rate throughout the acceleration phase. Thus the single-regime model gives a reasonable approximation when the vehicle is accelerating within a given gear. But they are greatly in error when the vehicle is accelerating up through the gears (Searle, 1999). A vehicle has higher acceleration at low speeds and lower acceleration at higher speeds. Additionally when the vehicle reaches top gear the acceleration is only a small fraction of what is available at lower speeds. However, this is not the case when single-regime model is used. The single-regime model thus, represents an approximation to the actual acceleration characteristics of vehicles. The speed and distance profiles represent an approximation as well.

\section{DUAL-REGIME MODEL}

To overcome the problems in the single-regime model, dual-regime model was formulated. The dual-regime model by using two different acceleration rates overcomes the problem in the single-regime model to an extent. The dual-regime model provides higher acceleration rate at lower speeds and lower acceleration rate at higher speeds. The model is logical, since it replicates the changing acceleration behavior of vehicles. The model basically implies that drivers are able to accelerate less after a certain speed is gained, since the engine is slower to generate more acceleration after a certain speed. This approach also provides better fit to the field data. Moreover, the model can be easily calibrated to fit field data compared to other acceleration models available in the literature. For details on formulation of the dual-regime model and comparison with other models, see (Bham and Benekohal, 2001a). The dual-regime model also provides realistic speeds with minimal calculation, making it feasible for use in traffic simulation models, since it requires minimal computational resources. The dual-regime model is incorporated in a traffic simulation model, CELLSIM (Bham and Benekohal, 2001b).

The two acceleration rates used in the dual-regime model can be found from the average speed profile of vehicles from the field data as shown in Figure 1. The acceleration rates can be calculated, similar to the way acceleration rates are calculated for the single-regime model. However, the speed versus time plot is divided into two different acceleration regimes. The first regime has a higher acceleration rate than the second. The second acceleration regime for the OSU and the FHWA data sets starts around a speed of $13 \mathrm{~m} / \mathrm{sec}$. The speed in the dual-regime model is updated as:

$$
\begin{aligned}
& v_{f}=v_{i}+a_{1} * t, 0 \leq v<13 \mathrm{~m} / \mathrm{sec}(29 \mathrm{mph}) \\
& v_{f}=v_{i}+a_{2} * t, v \geq 13 \mathrm{~m} / \mathrm{sec}(29 \mathrm{mph})
\end{aligned}
$$


where $a_{1}$ and $a_{2}$ are acceleration rates, $v_{f}$ and $v_{i}$ are final and initial speeds and $t$ equals time in seconds. They are presented in Table 1.

The acceleration constants for the first regime of the speed profile can be calculated using the time required to reach speed of approximately $13 \mathrm{~m} / \mathrm{sec}$. The acceleration rate for the second phase was obtained as the difference in speeds between the start and end of the second regime divided by the time interval. The ratio obtained for the first regime of 1.39 is similar to the ratio obtained for sedans of 1.32, discussed in the section on vehicle performance (Figure 3). The ratio of 1.32 was obtained by dividing the acceleration rates obtained for the years 1983 and 1968, same years the FHWA and OSU data sets were obtained.

In the absence of acceleration rates for a particular year, the ratios from the vehicle performance obtained using Figure 3 can be used to predict the vehicular performance for another year. For the dual-regime model the same can be done as well. However, the ratio for the second phase is different from the first phase, but in absence of information about the second acceleration phase, ratio from the first phase can be used. This is presented in Figure 4, where two different acceleration rates for the second regime were used for the FHWA data, one directly obtained from the data (0.86) and the other a value of $(0.58=1.39 * 0.42)$ obtained from the OSU data, see Table 1 for details. The acceleration rate obtained from the FHWA data for the second regime fits the field data better for obvious reasons. Figure 4 , also shows the closeness of fit of the dualregime to the field data for both the data sets.

\section{CONCLUSION}

The dual-regime model is more accurate in modeling the acceleration behavior of drivers in a platoon compared to the single-regime model. The speed profile from the dual-regime model provided better fit to the two field data sets used. Acceleration rates of 1.43 and $1.03 \mathrm{~m} / \mathrm{sec}$ were found for the FHWA and OSU, respectively, a ratio of 1.39 for the first regime. For the second regime, rates of 0.86 and 0.42 were found for the FHWA and OSU, respectively. Using the new approach described, the acceleration rate for the FHWA can be obtained from the OSU data by using the ratio of the first regime i.e. $1.39 * 0.42=0.58$. This was found to be close to the acceleration rate found for the FHWA data. Thus the approach works and provides good results. A speed of $13 \mathrm{~m} / \mathrm{sec}$ was found, around which the acceleration rate drops for the second phase. Using the approach, vehicle performance in the future can be predicted using the 0-60 mph data available for most vehicles in popular magazines. Improvement in vehicular performance can 
also be quantified using this approach. The acceleration capabilities of current vehicles can be adjusted to represent the performance of vehicles for past years, making older data collected, useful again. It is recommended that the approach should be tested for vehicle types other than sedans.

\section{REFERENCES}

1. Bham, G. H. and R. F. Benekohal (2001a). Development, Evaluation and Comparison of Acceleration Models in Traffic Simulation. Working paper.

2. Bham, G. H. and R. F. Benekohal (2001b). A High Fidelity Traffic Simulation Model based on Cellular Automata and Car-Following Concepts, Transportation Research Part C: Emerging Technologies, (submitted in review).

3. Searle, J. (1999). "Equations for Speed, Time and Distance for Vehicles under Maximum Acceleration." Advances in Safety Technology 1999, SP-1433, SAE, Inc., Warrendale, PA.

4. Smith, S. A., (1985). Freeway Data Collection for Studying Vehicle Interaction-Technical Report. Report No. FHWA/RD-85/108, U.S., Department of Transportation.

5. Treiterer, J. (1975). Investigation of Traffic Dynamics by Aerial Photogrammetry Techniques. Final Rep. EES278, Transportation Research Center, Ohio State University. 